\title{
Editorial
}

\section{The difficulties of assessing right ventricular function}

It is 20 years since Sade and Castaneda concluded from experiments investigating the effect of cautery of the canine right ventricular free wall ${ }^{1}$ and from the success of right ventricular bypass procedures for tricuspid atresia ${ }^{2}$ that the right ventricle is "dispensable". ${ }^{3}$ Since then the importance of right ventricular dysfunction in many cardiac conditions has become clearer, and the long-term results of the Fontan operation have suggested that the term "dispensable" may have been an oversimplification, because atrial arrhythmias and rising pulmonary impedance are often so poorly tolerated. In addition, it is increasingly apparent that right ventricular function is a significant factor in ischaemic heart disease.

Cohn et als description of the syndrome of right ventricular infarction, ${ }^{4}$ with raised venous pressure, low output, and clear lungs, suggested that the right ventricle may be of importance in coronary disease, and this was dramatically demonstrated in studies of acute inferior infarction reported by Zehender et al in which right ventricular infarction was associated with a $31 \%$ in hospital mortality and $64 \%$ major complication rate ${ }^{5}$ and in which thrombolysis was only of benefit in those patients with demonstrable right ventricular involvement. ${ }^{6}$ It has been proposed that right ventricular function may be a better guide than left ventricular function to functional state ${ }^{7}$ and even prognosis ${ }^{8}$ in patients with chronic heart failure caused by coronary disease. Furthermore, the assumption that right ventricular dysfunction is simply a marker for raised pulmonary resistance resulting from left ventricular dysfunction is challenged by the observation that right and left ventricular ejection fractions show no correlation in acute infarction and chronic heart failure. In Zehander et al's patients the left ventricular ejection fractions in groups with and without right ventricular infarction were the same. New techniques for studying ventricular function are therefore being applied to the right ventricle, but usually with disappointing results because it is difficult to assess right ventricular volume.

\section{Imaging}

Conventional imaging methods such as angiography, echocardiography, and radionuclide ventriculography, when combined with haemodynamic measurements, have given some insights into the relative importance of the various determinants of right ventricular pump function. Berger et al found that the radionuclide derived right ventricular ejection fraction correlated with the left ventricular ejection fraction, but not with right coronary stenosis. ${ }^{9}$ They concluded that raised afterload was the major cause of right ventricular pump failure. This was in keeping with a contemporary study of patients who had pulmonary hypertension with various non-respiratory causes,${ }^{10}$ which showed that even in those with conventionally recognised right ventricular failure, indices of contractile function such as $\mathrm{dP} / \mathrm{dt} \max$ and $V \max$ were indistinguishable from normal controls. Subsequent reports, however, suggest that both active and passive ventricular properties, and external factors can have important effects on overall function. Brown et al showed that the impaired right ventricular ejection fraction in patients with a raised wedge pressure was significantly worse in the presence of proximal right coronary disease. ${ }^{11}$ Berman et al showed that right ventricular end diastolic pressure was higher in patients with right coronary disease than in a matched group with significant pulmonary hypertension caused by aortic and mitral valve disease. ${ }^{12}$ Goldstein showed the importance of pericardial constraint and atrial dysfunction in patients with right ventricular infarction ${ }^{13}$ and in dogs with right coronary ligation..$^{14}$ It is clear, however, that in the study of the right ventricle, even more than the left, no technique that does not distinguish between the effects of afterload, contractility, ventricular compliance, and external constraint can adequately describe ventricular function.

\section{MRI scanning}

Magnetic resonance imaging (MRI) scanning has several advantages over other imaging techniques in the right ventricle. In 1986 Markiewicz et al showed that clear endocardial and epicardial signals from the right ventricle could be identified, and that there was a potential for volume estimation from tomographic cuts. ${ }^{15}$ More recently Suzuki et al used cine-MRI to study the right ventricle (estimating volume from $10 \mathrm{~mm}$ spaced simultaneous transverse slices analysed by Simpson's rule) and, by calculating an average of 18 volumes per cardiac cycle, constructed volume-time curves in patients with dilated ${ }^{16}$ and hypertrophic ${ }^{17}$ cardiomyopathy. On page 186 Naito et al describe the application of a presaturation tagging technique to the study of the right ventricle, and the study shows the advantages and weaknesses of MRI in this context. They confirm the expectation from echocardiographic and anatomical studies that the predominant contraction of the right ventricle is longitudinal and quantify that contraction regionally. However, neither this technique, describing regional shortening fraction, nor Suzuki et al's volume-time analysis allow the assessment of contractility or compliance that are the fundamentals of ventricular function. The most useful models for this kind of analysis in the left ventricle involve the description of pressure-volume relations within the cardiac cycle, at steady state and when loading varies. The time required to acquire one MRI study is a major obstacle to its application to the study of dynamic right ventricular pressure-volume relations.

Twenty years ago Suga et al described the active and passive properties of an isolated left ventricle as the constants that relate pressure to volume during systole and diastole. ${ }^{18}$ One success of this approach has been its ability to distinguish changes in contractility (Emax, preload recruitable stroke work) and compliance (stiffness) from changes in loading conditions and mechanical constraint, in an experimental and clinical setting. The other advantage of this model is that the viscoelastic properties of the 
ventricle and vascular bed into which it ejects can be described in common terms as an elastance (Emax) and effective arterial elastance (Ea), allowing a simple description of the coupling between the ventricle and its afterload. Maughan et al showed that the assumptions that underlie Suga et al's "time varying elastance" model are generally valid in the canine right ventricle, ${ }^{19}$ and the coupling between the right ventricle and the pulmonary circulation has been extensively investigated in animal models. In the pig, right ventricular stroke volume increased in response to increasing afterload until arterial elastance exceeded Emax, when falling stroke volume signalled right ventricular pump failure..$^{20}$

The ability to distinguish between the effects of loading and intrinsic changes in ventricular function makes pressure-volume analysis ideal for the study of right ventricular function in coronary disease and other causes of congestive heart failure. Conventional and new imaging techniques have as yet been unable adequately to provide a reliable, continuous right ventricular volume signal at a high enough acquisition frequency for the construction of continuous pressure-volume cycles, and it is likely that non-image dependent conductance catheter technology will provide the method for distinguishing between ventricular overload and ventricular failure in the future.

In conclusion, MRI provides a non-invasive method of assessing right ventricular volume at an acquisition frequency sufficient for the study of change during the cardiac cycle, and tagging adds the facility for a degree of regional wall motion analysis. Without the capacity for

1 Starr I, Jeffers A, Meade R. The absence of conspicuous increments of venous pressure after severe damage to the right ventricle of the dog,
with a discussion of the relation between clinical congestive failure and with a discussion of the relation between clinic
heart disease. Am Heart $\mathcal{f} 1943 ; 26: 291-301$.

2 Fontan F, Baudet E. Surgical repair of tricuspid atresia. Thorax 1971;26: 240-8.

3 Sade R, Castaneda A. The dispensable right ventricle. Surgery 1975;77: 624-31.

4 Cohn J, Guiha N, Broder M. Constantinos J. Right ventricular infarction. Am $\mathcal{F}$ Cardiol 1974;33:209-14.

5 Zehender M, Kasper W, Kauder E, Schonthaler M, Geibel A, Olschewski $M$, Just $H$. Right ventricular infarction as an independent predictor of prognosis after acute inferior myocardial infarction. $N$ Engl $f \mathrm{Med}$ 1993;328:981-8.

6 Zehender M, Kasper W, Kauder E, Schonthaler M, Geibel A, Olschewski $M$, Just $H$. Eligibility for and benefit of thrombolytic therapy in inferior $M$, Just $H$. Eligibility for and benefit of thrombolytic therapy in inferior
myocardial infarction: Focus on prognostic importance of right ventricmyocardial infarction: Focus on prognostic impo

7 Baker B, Wilen M, Boyd C, Dinh H, Franciosa J. Relation of right ventricular ejection fraction to exercise capacity in chronic left ventricular failure. Am ₹ Cardiol 1984;54:596-9.

8 Polak J, Holman L, Wynne J, Colucci W. Right ventricular ejection fraction: an indicator of increased mortality in patients with congestive heart failure associated with coronary artery disease. $7 \mathrm{Am}$ Coll Cardiol 1983;2:217-24.

9 Berger H, Johnstone D, Sands Y, Gottschalk A, Zaret B. Response of right ventricular ejection fraction to upright bicycle exercise in coronary artery disease. Circulation 1979;60:1292-300.

10 Stein P, Sabbah H, Anbe D, Marzilli M. Performance of the failing and nonfailing right ventricle of patients with pulmonary hypertension. $\mathrm{Am} \mathcal{F}$ Cardiol 1979;44:1050-5. simultaneous pressure recording and real time acquisition and display, however, it does not allow right ventricular function to be characterised in much more depth than the estimates of ejection fraction that are currently available from radionuclide techniques. It is well recognised that deteriorating right ventricular pump function may reflect the sensitivity of the right ventricle to changes in ventricular loading that may occur during left ventricular disease-directly through abnormal septal function and indirectly through increasing impedance in the pulmonary circulation. However, the high incidence of circulatory collapse independent of arrhythmia and more importantly of left ventricular function in patients with right ventricular involvement in acute inferior infarction suggests that right ventricular dysfunction is more than simply an index of left ventricular filling pressure. In Zehender et al's patients, those with infarction limited to the left ventricle had a mean left ventricular ejection fraction of $43 \%$ compared with $44 \%$ in the group with right ventricular involvement. Despite this, $27 \%$ of the patients in which the right ventricle was affected developed cardiogenic shock compared with $5 \%$ of those with simple left ventricular infarction. The right ventricle can therefore no longer safely be assumed to be following its dominant partner. It requires independent assessment and perhaps treatment in many patients.

PAUL OLDERSHAW ANDREW BISHOP

Royal Brompton Hospital,

Sydney Street,

London SW3 $6 \mathrm{NP}$

11 Brown K, Okada R, Boucher C, Strauss W, Pohost G. Right ventricular ejection fraction response to exercise in patients with coronary artery disease: influence of both right coronary artery disease and exercise induced changes in right ventricular afterload. $\mathcal{f} \mathrm{Am}$ Coll Cardiol 1984; induced chang

12 Berman J, Green L, Grossman W. Right ventricular diastolic pressure in coronary artery disease. $A m \mathcal{F}$ Cardiol $1979 ; 44: 1263-8$.

13 Goldstein J, Barzilai B, Rosamond T, Eisenberg P, Jaffe A. Determinants of haemodynamic compromise with severe right ventricular infarction. Circulation 1990;82:359-68.

14 Goldstein J, Vlahakes G, Verrier E, Schiller N, Tyberg J, Ports T, Parmley tion and elevated intrapericardial pressure in the genesis of low outpu

15 Markiewicz W, Sechtem U, Higgins C. Evaluation of the right ventricle by magnetic resonance imaging. Am Heart f 1987;113:8-15.

16 Suzuki J, Caputo G, Masui T, Chang J, O'Sullivan M, Higgins C. Assessment of right ventricular diastolic and systolic function in patients with dilated cardiomyopathy using cine magnetic resonance imaging. Am Heart f 1991;122:1035-40.

17 Suzuki J, Chang J, Caputo G, Higgins C. Evaluation of right ventricular early diastolic filling by cine nuclear magnetic resonance imaging in patients with hypertrophic cardiomyopathy. $\mathcal{F}$ Am Coll Cardiol 1991;18 $120-6$.

18 Suga H, Sagawa K, Shoukas A. Load independence of the instantaneous pressure-volume ratio of the canine left ventricle and the effects of epinephrine and heart rate on the ratio. Circ Res 1973;32:314-22.

19 Maughan M, Shoukas A, Sagawa K, Weisfeldt M. Instantaneous pressure-volume loop of the canine right ventricle. Circ Res 1979;44:309-15.

20 Fourie P, Coetzee A, Bolliger C. Pulmonary artery compliance: its role in ventricular-arterial coupling. Cardiovasc Res 1992;26:839-44. 